\title{
Activation of frozen ferroelectric domain wall by magnetic field sweeping in a multiferroic $\mathrm{CuFeO}_{2}$
}

\author{
H. Tamatsukuri, ${ }^{1, *}$ S. Mitsuda, ${ }^{1}$ T. Nakajima, ${ }^{1}$ K. Shibata, ${ }^{1}$ C. Kaneko, ${ }^{1}$ K. Takehana,${ }^{2}$ Y. Imanaka, ${ }^{2}$ N. Terada,${ }^{3}$ \\ H. Kitazawa, ${ }^{3}$ K. Prokes, ${ }^{4}$ S. Matas, ${ }^{4}$ K. Kiefer, ${ }^{4}$ S. Paeckel, ${ }^{4}$ A. Sokolowski, ${ }^{4}$ B. Klemke, ${ }^{4}$ and S. Gerischer ${ }^{4}$ \\ ${ }^{1}$ Department of Physics, Faculty of Science, Tokyo University of Science, Tokyo 162-8601, Japan \\ ${ }^{2}$ National Institute for Materials Science, Nano Physics Group, 3-13 Sakura, Tsukuba, Ibaraki 305-0003, Japan \\ ${ }^{3}$ National Institute for Materials Science, Neutron Scattering Group, 1-2-1 Sengen, Tsukuba, Ibaraki 305-0047, Japan \\ ${ }^{4}$ Helmholtz-Centre Berlin for Materials and Energy, Glienicker Straße 100, Berlin 14109, Germany
}

(Dated: October 13, 2015)

\begin{abstract}
In a ferroelectric helimagnetic phase of a spin-driven multiferroic $\mathrm{CuFeO}_{2}$, we have found irreversibly additive evolution of electric polarization $(P)$ induced by sweeping magnetic field $(H)$ under an applied electric field $(E)$, despite a large coercive electric field in the phase. From the unpolarized neutron diffraction experiments with $i n$-situ $P$ measurements under applied $E$, we have revealed that increment of $P$ is achieved by the variation of an incommensurate magnetic modulation wave number $(q)$ of the helical magnetic ordering in $H$-sweeping regardless of increasing or decreasing $H$. Combining this result with $H$ dependence of the magnetic diffraction intensity and a result of off-bench $P$ measurements, we conclude that the $H$ evolution of $P$ is caused by a change in a (ferroelectric) helicity domains volume fraction by driving the helicity domain wall (DW). Taking into account the results of further detailed $P$ measurements, we propose a possible model of the phenomenon that, instead of simply driving the helicity DW, the variation of $q$ causes the neighborhood of the helicity DWs to store an extra exchange energy and to be sensible for $E$, and then, these regions and the helicity DWs are reconstructed by applied $E$ so as to enlarge the helicity domain favored by the direction of $E$. The present study demonstrates the magneto-electric cross correlation in driving multiferroic DW: we could activate the frozen ferroelectric DW by means of $H$-sweeping. This is also an achievement of driving an antiferromagnetic DW, which is difficult in conventional antiferromagnets in principle.
\end{abstract}

\section{INTRODUCTION}

In recent years, many magnetic materials with "spindriven ferroelectricity" have been reported. ${ }^{1-4}$ These materials, known as multiferroics, show a giant magnetoelectric (ME) effect, which is the induction or control of electric polarization by a magnetic field $(H)$, and vice versa. In these materials, the ferroelectricity often emerges as a result of inversion-symmetry breaking by spiral magnetic structures such as a cycloid or a proper screw. As for microscopic mechanism how these magnetic structures induce the ferroelectric polarization $(P)$, several theories have been proposed and well explained giant ME effect observed for such spin-driven ferroelectric materials. ${ }^{5-7}$ In general, however, bulk properties depend not only on microscopic magnetic structures but also on semi-maroscopic multiferroic domain structures or domain-wall (DW) structures, which are expected to show a novel response to external fields. For example, multiferroic DW motion caused by AC electric field $(E)$ accounts for magnetically tunable dielectric dispersions in $\mathrm{DyMnO}_{3} \cdot{ }^{8,9}$ Also, from the viewpoint of an application of multiferroic devices such as non-volatile memory, ${ }^{10}$ it is necessary to investigate the multiferroic domain structures or DW structures as well as magnetic structures.

Delafossite compound $\mathrm{CuFeO}_{2}$ (CFO) system investigated here is one of the model materials exhibiting the spin-driven ferroelectricity. CFO is known as a typical example of trianglular-lattice antiferromagnets, in which magnetic moment is carried by $\mathrm{Fe}^{3+}$ ion $(S=5 / 2)$.
In zero $H$, successive magnetic phase transitions occur from the paramagnetic (PM) phase to the collinear 4sublattice (4SL) phase $(\uparrow \uparrow \downarrow \downarrow)\left(T \leq T_{\mathrm{N} 2} \sim 11 \mathrm{~K}\right)$ with the magnetic moments along the $c$ axis, through a partially disordered $(\mathrm{PD})$ phase $\left(T_{\mathrm{N} 2} \leq T \leq T_{\mathrm{N} 1} \sim 14 \mathrm{~K}\right)$ with a sinusoidally amplitude-modulated magnetic structure having moments along the $c$ axis. ${ }^{11}$ When $H$ is applied along the $c$ axis up to $15 \mathrm{~T}$ below $T_{\mathrm{N} 2}$, two other magnetic phases are realized: ${ }^{12,13}$ a ferroelectric-incommensurate magnetic (FE-ICM) phase with a helical magnetic structure $(7 \mathrm{~T} \lesssim H \lesssim 12 \mathrm{~T})$ and a collinear 5 -sublattice (5SL) phase ( $\uparrow \uparrow \downarrow \downarrow)$ with magnetic moments along the $c$ axis $(H \geq \sim 12 \mathrm{~T})$. The FE-ICM phase is also realized even under zero $H$ by substituting a few percent of nonmagnetic ions $\left(\mathrm{Al}^{3+}, \mathrm{Ga}^{3+}\right.$, or $\left.\mathrm{Rh}^{3+}\right)$ for $\mathrm{Fe}^{3+} .{ }^{14-17}$

In all of these phases, the magnetic modulation vector is $(q, q, 3 / 2)$. Because of the trigonal symmetry along the $c$ axis, CFO has the other two magnetic modulation vectors of $(-2 q, q, 3 / 2)$ and $(q,-2 q, 3 / 2)$, which are equivalent to $(q, q, 3 / 2)$, resulting in an existence of three magnetic domains. We refer to them as (110), (210) and $(1 \overline{2} 0)$ domains, respectively, and collectively call $q$ domains. In the FE-ICM phase, helical magnetic structure whose screw axis is parallel to [110] (or [210],[120]) direction induces ferroelectric polarizations parallel to [110] (or $[\overline{2} 10],[1 \overline{2} 0]$ ) direction, ${ }^{18,19}$ which arise through $d-p$ hybridization mechanism. ${ }^{7}$ Because there are one-to-one correspondence between directions of $P$ and the spin helicity (a right-handed or left-handed helical arrangement of spins), ${ }^{19}$ each $q$ domain consists of two domains hav- 
ing different helicity. We refer to them as helicity domains. Consequently, six multiferroic domains exist in the FE-ICM phase of CFO as shown in Fig. 1(a). By taking into account the magnetic domain structure and magnetic structure in the FE-ICM phase, $P_{[110]}$, which is $P$ macroscopically measured by using [110] electrodes, is determined by three factors: (i) the $q$ domains volume fractions, (ii) the helicity domains volume fractions, (iii) the local electric polarization determined by the helical magnetic structure $\left(P_{\text {local }}\right)$. When we use the [110] electrodes, $P$ in $(\overline{2} 10)$ or $(1 \overline{2} 0)$ domains contributes only a half to $P_{[110]}$, because it is projected onto the [110] electrodes by $\cos 60^{\circ}$ as shown in Fig. 1(a). This is the reason why the value of $P_{[110]}$ is affected also by the change in the $q$ domains volume fractions.

Since CFO systems form such six-multiferroic domain structure in the FE-ICM phase, novel control of the multiferroic domains volume fraction have been performed. For example, Nakajima et al. reported that the increase or decrease of $P$ in the FE-ICM phase of $\mathrm{CuFe}_{1-\mathrm{x}} \mathrm{Ga}_{\mathrm{x}} \mathrm{O}_{2}$ is synchronized with an uniaxial pressure via the lattice distortion. ${ }^{20}$ Seki et al. reported that $H$ rotating within the $a b$ plane causes $120^{\circ}$ flop of $P$ in the FE-ICM phase of $\mathrm{CuFe}_{1-\mathrm{x}} \mathrm{Ga}_{\mathrm{x}} \mathrm{O}_{2} .{ }^{21}$ These studies were achieved by the reconstruction of $q$ domain structures below the ferroelectric phase transition temperature. In contrast, the uniaxial pressure is useless for the reconstruction of helicity domain structures below the ferroelectric phase transition temperature in this system, because the helicity domains have no coupling with the lattice distortion in this system. Also with regard to the magnetic filed, for directly driving the helicity DWs, we need to apply the conjugate magnetic field to the order parameter in this system (namely, the magnetic field having the helical magnetic modulation wave vector in the FE-ICM phase). Consequently, only $E$ remains as the practicable external field for driving the helicity DWs. Nevertheless, both the $q$ DWs and the helicity DWs are not driven by $E$ owing to a large coercive electric field above $1 \mathrm{MV} / \mathrm{m}$ at the low temperature in this system, even though the (a)

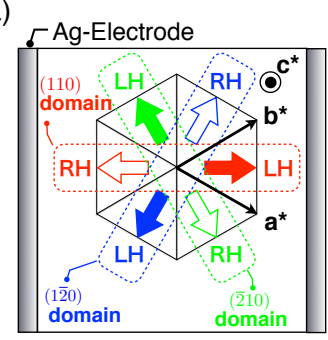

(b)

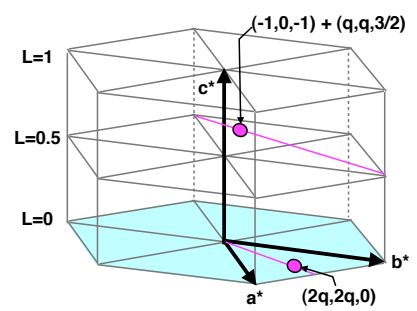

FIG. 1. (Color Online) (a) Schematic illustration of 6 magnetic domains and experimental configuration for measurements of $P$. (b) Locations of the magnetic Bragg points in the reciprocal lattice space. For visibility, the ratio of $c^{*}$ to $a^{*}\left(\right.$ or $\left.b^{*}\right)$ is modified. nuclei growth of one of the helicity domains at (the first order) ferroelectric transitions can be easily promoted by an applied $E .^{22}$ Thus, the reconstruction of helicity domain structures below the ferroelectric phase transition temperature has not been accomplished yet in this system.

In this paper, we report a peculiarly irreversible $H$ evolution of $P$ induced by $H$-sweeping under applied $E$ in the FE-ICM phase of CFO, which is caused by a change in the helicity domains volume fractions by driving the helicity DWs. Our study demonstrates that a $H$ variation of the incommensurate wave number $q$ can activate the frozen ferroelectric helicity DWs due to the large coercive electric field at temperatures well below the ferroelectric phase transition temperature $\sim 9 \mathrm{~K}$. This is also an achievement of driving antiferromagnetic DW through the cross correlation between antiferromagnetism and ferroelectricity. For this phenomenon, we propose a model including a mechanism of driving the helicity DWs, which is expected to be realized in other spin-driven ferroelectrics as well.

\section{EXPERIMENTAL DETAILS}

Single crystals of $\mathrm{CFO}$ and $\mathrm{CuFe}_{1-\mathrm{x}} \mathrm{Al}_{\mathrm{x}} \mathrm{O}_{2}(\mathrm{x}=0.02)$ (CFAO) of nominal compositions were prepared by the floating zone method. ${ }^{23}$

\section{A. Electric polarization measurements}

For the $P_{[110]}$ measurements, crystals were cut into thin plates $\left(\sim 3 \times 4 \times 0.26 \mathrm{~mm}^{3}\right)$ with the widest faces perpendicular to the [110] direction. As the electrodes, silver paste was painted onto these surfaces, and $E$ (typically, $800 \mathrm{kV} / \mathrm{m}$ ) was applied along the [110] axis. $H$ was applied along the $c$ axis using 15T superconducting magnet installed at Tsukuba Magnet Laboratory in National Institute for Materials Science (NIMS). $P$ was deduced by the time integration of a polarization current (typical peak values are $\sim 1 \times 10^{-11} \mathrm{~A}$ at $2 \mathrm{~K}$ or $\sim 2.5 \times 10^{-11}$ A at $7 \mathrm{~K}$ ) flowing through changing in temperature $(T)$ or $H$, which was measured with an electrometer (Keithley $6517 \mathrm{~A})$. The typical sweep rate for $H$ was $11.4 \mathrm{mT} / \mathrm{s}$. When $E$ was applied, a leakage current flowed (for example $\sim 9 \times 10^{-14}$ A at $2 \mathrm{~K}$ or $\sim 1 \times 10^{-12}$ A at $7 \mathrm{~K}$ ) because $\mathrm{CFO}$ and CFAO are intrinsic semiconductors. We thus subtracted it from the polarization current before time integration. Unless specifically noted, the data of $P_{[110]}$ were obtained at NIMS.

\section{B. Neutron diffraction experiments}

Neutron diffraction measurements were carried out at the two-axis diffractometer E4 at Helmholtz-Zentrum Berlin (HZB). The wavelength of incident neutron was 
$2.44 \AA$. $H$ was applied using a $14.5 \mathrm{~T}$ vertical field cryomagnet VM-1. We employed two diffraction setups for simultaneous measurements of $H$ dependence of $P_{[110]}$ and $q$ and for measurements of $2 q$.

\section{Neutron diffraction experiments with in-situ electric polarization measurements}

For the neutron diffraction experiments with in-situ $P_{[110]}$ measurements, the crystal was cut into thin plate $\left(4.26 \times 5.91 \times 1.06 \mathrm{~mm}^{3}\right)$ with the widest faces perpendicular to the [110] axis. The electrodes consisted of silver paste painted onto these surfaces. $P$ was deduced by the same manner as described above. The polarization currents were measured with an electrometer (Keithley 6517B). Here, we should mention that measurements of polarization currents in these experiments were rather problematic. Because the sample used in the experiments was relatively thick to gain the neutron counts, we needed to apply a high voltage $(V)$ of approximately $800 \mathrm{~V}$ to the sample in order to adapt the magnitude of $E$ to the data obtained at NIMS. When the high voltage, from $400 \mathrm{~V}(E \simeq 377 \mathrm{kV} / \mathrm{m})$ to $1000 \mathrm{~V}(E \simeq 943 \mathrm{kV} / \mathrm{m})$, was applied to the sample, however, we observed an unexpected current, which flowed in the inverse direction to signal polarization current (Fig. 3(c)). This nuisance current was conspicuously seen at higher temperatures, especially at $8.5 \mathrm{~K}$, and we could not deduce $P_{[110]}$ at that temperature. An origin of this nuisance current has not yet been determined. When we restricted applied voltage to below $400 \mathrm{~V}$, however, we could obtain the current data without the nuisance current, which qualitatively reproduces the value of $P_{[110]}$ observed previously in $P_{[110]}$ measurements at NIMS.

To provide access to the FE-ICM phase, we need to apply vertical $H$ along the $c$ axis, which means that we can only survey magnetic reflections with spin density projected on the $c$ plane in the $(h k 0)$ zone. In addition, the only accessible fundamental $(q, q, 0)$ magnetic Bragg reflections are forbidden, because the helical spin arrangements in the $c$ plane are stacked antiferromagnetically along the $c$ axis. ${ }^{12}$ For this reason, we measured the $H$ dependence of $q$ using $(\overline{1}, 0, \overline{1})+(q, q, 3 / 2)$ fundamental magnetic Bragg reflections by inclining the sample from the $c$ axis (by $\sim 7^{\circ}$ ) for vertical $H$ (see Fig. 1(b)). Note that the $(\overline{1}, 0, \overline{1})+(q, q, 3 / 2)$ magnetic Bragg point belongs to the (110) domain. Moreover, $P_{[110]}$ measured in this experiment is mainly attributed to $P$ in the (110) domain, because poling electric field act only a half to $(\overline{2} 10)$ or $(1 \overline{2} 0)$ domains due to the [110] electrodes, and besides, $P$ in $(\overline{2} 10)$ or $(1 \overline{2} 0)$ domains contributes only a half to $P_{[110]}$ as described in Sec. I. Therefore, the reflections are suitable for simultaneous measurements of $q$ and $P_{[110]}$. In principle, we need to perform $(h-1, h, 0.5)$ reciprocal lattice scan in order to follow a $H$ variation of the $(q, q, 3 / 2)$ magnetic Bragg point. However, this is difficult in the experimental configuration. Therefore, we have performed $\omega$ scan using two-dimensional E4 area detector, which covers a wide range of $2 \theta$. Although we can not deduce the absolute value of $q$ from this scan due to lack of observable Bragg reflections as a reference, we can detect a peak shift in the scan corresponding to a variation of $q$ as shown in Fig. 3(a). We have then linearly mapped the peak shift in $\omega$ scan to a $H$ dependence of $q$ using $H$ dependence of $2 q$ as described below. We have confirmed that the nature of the $H$ dependence of $P_{[110]}$ does not change even by applying slightly inclined $H$, although the phase transition field was slightly modified. The typical rate of $H$-sweeping was $8.67 \mathrm{mT} / \mathrm{s}$.

\section{Measurements of $2 q$}

As described above, fundamental $(q, q, 0)$ magnetic Bragg reflections are forbidden because of the antiferromagnetic stacking of the helical magnetic orderings along the $c$ axis. Therefore, to obtain the absolute value of $q$, we have measured the $H$ dependence of $2 q$ using the $(2 q, 2 q, 0)$-second-harmonic reflections (see Fig. 1(b)) arising from the distortion of the helical magnetic structure in regular setup. This non-inclined setup enable us to perform reciprocal lattice scan along $(h, h, 0)$ line. Because we can measure the magnetic reflections from $(-4 q, 2 q, 0)$ or $(2 q,-4 q, 0)$ magnetic Bragg points belonging to the other $q$ domains, we can also directly survey the $H$ change in the $q$ domains volume fractions from this experiments. For these measurements, we have used a relatively large sample $(\sim 1 g)$. Note that $E$ was not applied in this experiment.

\section{RESULTS AND DISCUSSION}

A. $H$ evolution of $P$ by the $H$ variation of the incommensurate magnetic modulation wave number $q$ in the FE-ICM phase

Figure 2(c)[i] shows the $H$ dependence of $P_{[110]}$, which is deduced from the polarization current obtained by increasing or decreasing $H$ from $10 \mathrm{~T}$ at $2 \mathrm{~K}$. Before the measurement, poling electric field $\left(E_{p}\right)$ of $800 \mathrm{kV} / \mathrm{m}$ was applied typically at $16 \mathrm{~K}$ on cooling, which is well above the ferroelectric phase transition temperature $\sim 9 \mathrm{~K}$, and was removed at $2 \mathrm{~K}$, as shown in Fig. 2(a)[i]. Hereafter, we refer to the application of $E_{p}$ through the phase transition as proper poling. By proper poling, we can obtain the macroscopic $P_{[110]}$ as a result of an imbalance of the helicity domains volume fraction. On the other hand, when we apply $E$ of $800 \mathrm{kV} / \mathrm{m}$ at $2 \mathrm{~K}$ (well below the phase transition temperature) after cooling without $E_{p}$ at $10 \mathrm{~T}, P_{[110]}$ showes almost no macroscopic change and remaines at $0 \mathrm{C} / \mathrm{m}^{2}$. This indicates that the six multiferroic domains volume fractions does not change even when $E \sim 800 \mathrm{kV} / \mathrm{m}$ is applied in the FE-ICM phase and the domain walls have a large coercive electric field. 


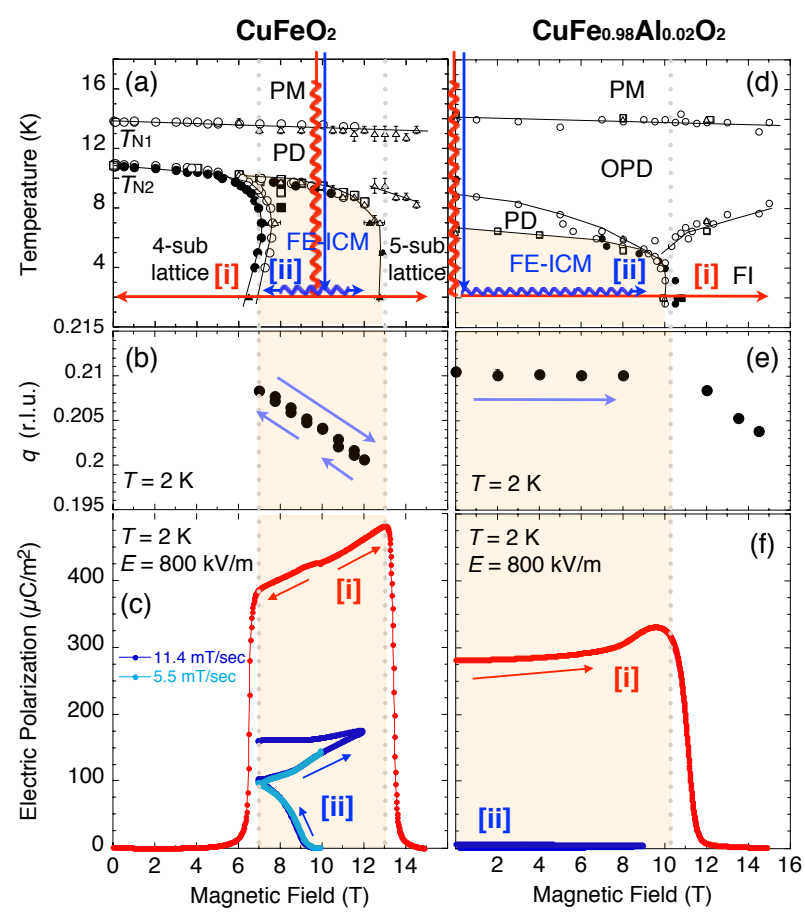

FIG. 2. (Color Online) (a) Measurement paths on $H-T$ phase diagram for $\mathrm{CuFeO}_{2}$. Wavy lines denote that electric field is applied. In process [i], the polarization current was measured with increasing $H$ to $15 \mathrm{~T}$ or decreasing $H$ to $0 \mathrm{~T}$ from $10 \mathrm{~T}$ at $2 \mathrm{~K}$ without $E$. Before the measurements, $E_{p}$ was applied typically at $16 \mathrm{~K}$ on cooling, and was removed after cooling down to $2 \mathrm{~K}$. In process [ii], the polarization current was measured with $H$-sweeping in the FE-ICM phase under applied $E$ at 2 $\mathrm{K}$ after cooling without $E_{p}$ at $10 \mathrm{~T}$. [(b)-(c)] Magnetic field dependence of (b) $q$, (c) $P_{[110]}$ (with 2 kinds of $H$-sweeping rate for process [ii]) at $2 \mathrm{~K}$ in $\mathrm{CuFeO}_{2}$. (d) Measurement paths on $H-T$ phase diagram for $\mathrm{CuFe}_{0.98} \mathrm{Al}_{0.02} \mathrm{O}_{2}$. Wavy lines denote that electric field is applied. [(e)-(f)] Magnetic field dependence of (e) $q$, (f) $P_{[110]}$ at $2 \mathrm{~K}$ in $\mathrm{CuFe}_{0.98} \mathrm{Al}_{0.02} \mathrm{O}_{2}$. The data of (a) and the data of (d),(e) are taken from Ref. 12 and 24 , respectively.

However, we have found a peculiar emergence of $P_{[110]}$ by $H$-sweeping from $10 \mathrm{~T}$ to $7 \mathrm{~T}$ with applied $E$ of 800 $\mathrm{kV} / \mathrm{m}$ as shown in Fig. 2(a)[ii] and 2(c)[ii]. Remarkably, when $H$ is subsequently swept from $7 \mathrm{~T}$ to $12 \mathrm{~T}$ under applied $E$, this emerged $P_{[110]}$ irreversibly increases, instead of reversibly decreasing. For further $H$-sweeping from $12 \mathrm{~T}$ to $7 \mathrm{~T}, P_{[110]}$ additively evolves.

To investigate a mechanism of this phenomenon, we have focused on a magnetic modulation wave number $q$ in the FE-ICM phase as a physical quantity having a $H$ dependence $^{12}$ (see Fig. 2(b) ${ }^{25}$ ) and have performed similar experiments using CFAO. As shown in Figs. 2(d) and 2(e), CFAO exhibits the FE-ICM phase even under zero $H,{ }^{14,15}$ and $q$ is almost field independent. ${ }^{24} \mathrm{By}$ proper poling, CFAO shows the same order of magnitude of $P_{[110]}$ in the FE-ICM phase as does CFO (Fig. $2(\mathrm{~d})[\mathrm{i}]$ and $2(\mathrm{f})[\mathrm{i}])$, whereas $P_{[110]}$ does not emerge by
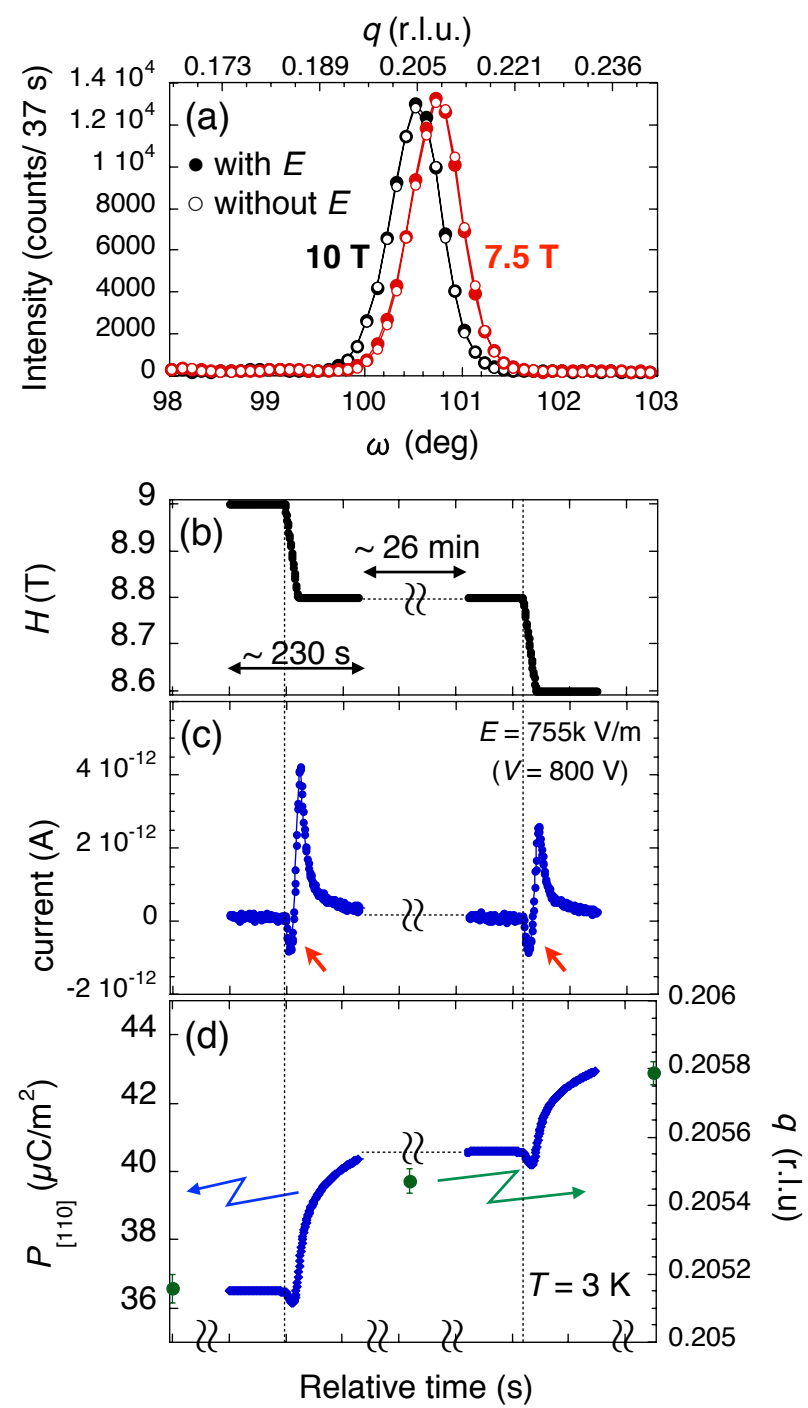

FIG. 3. (Color Online) (a) Typical diffraction profiles at $10 \mathrm{~T}$ and $7.5 \mathrm{~T}$ with and without applied $E$ at $3 \mathrm{~K}$. Upper abscissa shows $q$ value corresponding to the angle shown in lower abscissa. These $q$ values are obtained by the linear mapping using the result of $2 q$ measurements, as mentioned in Sec. II B 1. [(b)-(d)] Typical results of the neutron diffraction experiments with in-situ $P$ measurements from 9 to $8.6 \mathrm{~T}$ at $3 \mathrm{~K}$. The change in (b) $H$, (c) polarization current, and (d) $P_{[110]}$ and $q$ as a function of relative time. The data of polarization current and $P_{[110]}$ in this figure were obtained at HZB. Red arrows in Fig. 3(c) indicate the nuisance current due to applied voltage $V$ of $800 \mathrm{~V}$.

$H$-sweeping under applied $E$ in contrast to CFO (Fig. 2(d)[ii] and 2(f)[ii]). These results indicate that the $H$ variation of $q$ plays a key role in this phenomenon.

Thus, by neutron diffraction experiments with in-situ $P_{[110]}$ measurements, we have investigated a relationship between the $H$ evolution of $P_{[110]}$ and that of $q$, and specified an origin of this phenomenon among three factors (i) $\sim$ (iii) for determining $P_{[110]}$ as described in Sec. I. First 


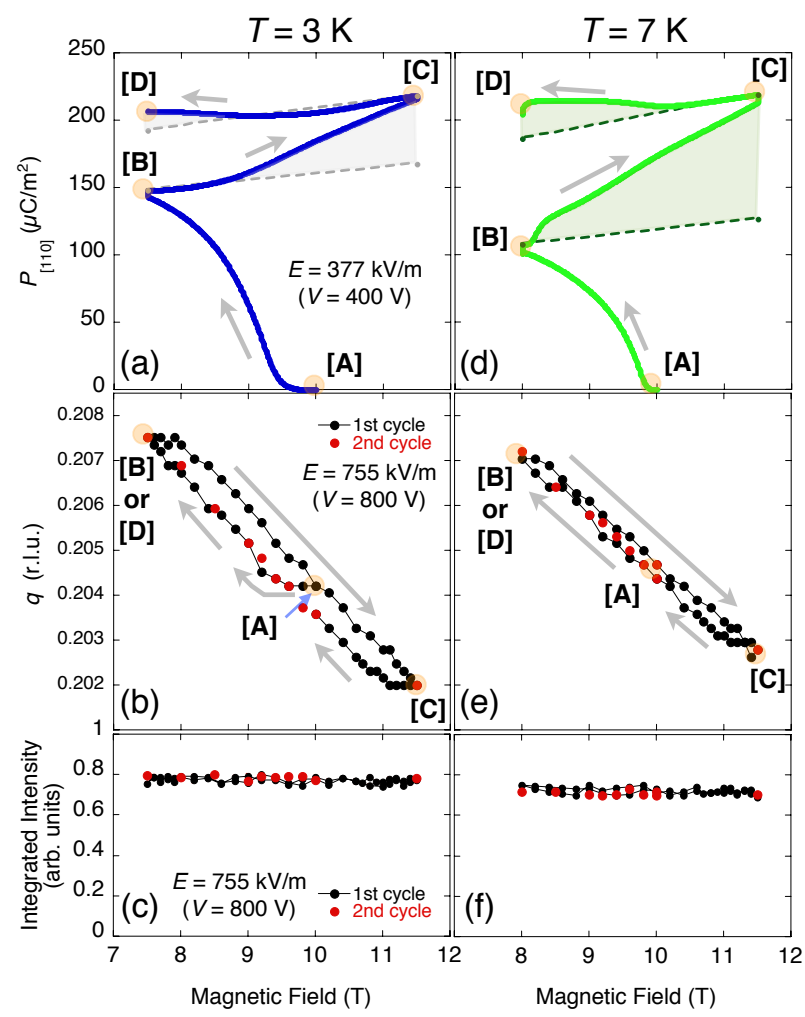

FIG. 4. (Color Online) [(a)-(c)] $H$ evolution of (a) $P_{[110]}$, (b) $q$ and (c) integrated intensity under applied $E$, starting from un-polarized state prepared by cooling down to $3 \mathrm{~K}$ without $E_{p}$ at 10 T. [(d)-(f)] $H$ evolution of (d) $P_{[110]}$, (e) $q$ and (c) integrated intensity under applied $E$ at $7 \mathrm{~K}$. Gray or green dashed lines are guide lines to demonstrate the $H$ dependence of $P_{[110]}$ obtained by scaling the data measured with proper poling procedure (see text for details). The data of $P_{[110]}$ in this figure were obtained at HZB.

of all, as shown in Fig. 3(a), we have confirmed that the $H$ dependence of $q$ is the same under applied $E \neq 0$ or $E=0$. Next, we show the outline of the neutron diffraction experiments with in-situ $P_{[110]}$ measurements. As shown in Fig. 3(b)-3(d), after the value of $q$ was measured at $9 \mathrm{~T}$ (Fig. $3(\mathrm{~d})$ ), we swept $H$ from 9 to $8.8 \mathrm{~T}$ (Fig. 3(b)) while measuring polarization currents (Fig. $3(\mathrm{c}))$. Then, the value of $q$ was measured again at $8.8 \mathrm{~T}$, and subsequently, we again swept $H$ from 8.8 to $8.6 \mathrm{~T}$ while measuring polarization currents. By step-by-step $H$-sweeping in such a way, we have obtained the $H$ dependence of $q$ and that of $P$, as shown in Fig. 3(d). Note that we have measured the polarization current only in the middle of $H$-sweeping, and thus we do not obtain the current data during each measurements of $q$. Moreover, even after stopping $H$-sweeping, the $P_{[110]}$ gradually increases through a relaxation process, as described in section III C in details. Therefore, the absolute value of $P_{[110]}$ is not accurately determined by this simultaneous measurements with the step-by-step $H$-sweeping. In addition, the observed currents include the nuisance currents due to applied voltage of $800 \mathrm{~V}$ as described in Sec. II B 1 (as indicated by red arrows in Fig. 3(c)). Therefore, the accuracy of the absolute value of $P_{[110]}$ is limited. In Figs. 3(c) and 3(d), we show typical results of polarization currents, $P_{[110]}$, and $q$ as a function of relative time, obtained by step-by-step $H$-sweeping from 9 to 8.6 $\mathrm{T}$ at $3 \mathrm{~K}$. Despite the inaccurate absolute values of $P_{[110]}$, these data distinctly demonstrate that $P_{[110]}$ begins to increase when $H$ starts to vary, and correspondingly, the value of $q$ is slightly but notably changed before and after the $H$-sweeping.

To discuss the origin of this phenomenon and the relationship between the $H$ evolution of $P_{[110]}$ and that of $q$ in more detail, we show the $H$ evolution of $P_{[110]}$, that of $q$ and the $H$ dependence of integrated intensity under applied $E$, starting from un-polarized state prepared by cooling down to $3 \mathrm{~K}$ at $10 \mathrm{~T}$ without $E_{p}$ in Figs. 4(a)4(c). Note that, for clarity in Fig. 4(a) (and Fig. 4(d)), we show the result of $P_{[110]}$ measured by continuously $H$ sweeping, instead of the step-by-step $H$-sweeping. Here, we have measured $P_{[110]}$ in off-bench experiments at HZB immediately after the neutron beam experiments keeping the experimental setup at the low $T$, and restricted applied voltage to $400 \mathrm{~V}(E \simeq 377 \mathrm{kV} / \mathrm{m})$ to avoid the nuisance current. As it will be shown later (see Fig. $7(\mathrm{a})$ ), we have confirmed that the overall $H$ evolution of $P_{[110]}$ measured by the continuous $H$-sweeping is intrinsically equivalent to one measured by the step-by-step $H$-sweeping. As shown in Fig. 4(c) and 4(f), the $H$ dependence of integrated intensity of $(q-1, q, 0.5)$ reflection does not change over several cycles of $H$-sweeping even under applied $E$. Because unpolarized neutron was used in this experiments, the helicity domains volume fractions can not be discriminated from the magnetic reflections, which means that the integrated intensity of $(q-1$, $q, 0.5)$ reflection is proportional to one of the $q$ domains volume fraction, namely, (110) domain in this experimental setup. This result indicates that the $q$ domain walls are not driven by $H$-sweeping or by application of $E$, and that the $q$ domains volume fractions do not change. Therefore, the factor (i) the $q$ domains volume fractions described in Sec. I is not the origin of the $H$ evolution of $P_{[110]}$. Although the $q$ domains volume fractions can be changed by $H$ rotating within the $a b$ plane, as reported by Seki et al. ${ }^{21}$ the origin of this phenomenon is entirely different from reconstruction of $q$ domain structures.

Next, we consider a $H$ variation of $P_{\text {local }}$ (the factor (iii)) in the $H$ evolution of $P_{[110]}$. As was reported by Nakajima et al. ${ }^{26}$ by proper poling with applied $E_{p} \geq \sim$ $600 \mathrm{kV} / \mathrm{m}, P$ originating from an imbalance of the helicity domains volume fractions (the factor (ii)) is saturated in $P-E_{p}$ curve (see FIG. 10 inset in ref. 26). The helicity domains volume fractions are thus considered to be close to 1 . In other words, when we perform proper poling, $P$ originating from the imbalance of the helicity domains volume fractions is not increased by $H$-sweeping. Therefore, by taking into account the fact that the $q$ domains 
volume fractions do not change by $H$-sweeping, the linear $H$ dependence of $P_{[110]}$ obtained by proper poling procedure (Fig. 2(c)[i]) is considered to come from the $H$ variation of $P_{\text {local }}$ determined by the helical magnetic structure. Thus, the peculiarly irreversible $H$ evolution of $P_{[110]}$ includes the $H$ variation of $P_{\text {local }}$ with the linear $H$-dependence, which is reversible, during $H$-sweeping, although the $H$ variation of the factor (iii) $P_{\text {local }}$ is not the origin of the $H$ evolution of $P_{[110]}$.

For the reasons stated above, deviation from the linear $H$ variation of $P_{\text {local }}$ is caused by a change in the factor (ii) the helicity domains volume fractions. To demonstrate how the $H$ evolution of $P_{[110]}$ includes the linear $H$ variation of $P_{\text {local }}$, we plot gray dashed guide lines as shown in Fig. 4(a). These guide lines represent the $H$ dependence of $P_{[110]}$ expected by only the $H$ variation of $P_{\text {local }}$ in a process from point [B] to point [C] (or a process from point $[\mathrm{C}]$ to point $[\mathrm{D}])$. As it is clearly seen in the process from point $[\mathrm{B}]$ to point $[\mathrm{C}]$ in Fig. 4(a), $P_{[110]}$ follows the dashed line at the beginning, and then starts to deviate from the dashed line upward and evolve additively. These results indicate that the peculiar $H$ evolution of $P_{[110]}$ is attributed to the change in the helicity domains volume fractions, which is achieved by driving the helicity DWs. In addition, corresponding to the behavior of $P_{[110]}, q$ is almost constant for a while close to the point $[\mathrm{B}]$, and then begins to vary, as shown in Fig. 4(b). Similarly, in the process from point $[\mathrm{C}]$ to point $[\mathrm{D}], P_{[110]}$ decreases at the beginning instead of monotonically increasing, following the linear $H$ variation of $P_{l o c a l}$, and then deviates from the dashed line and increases. Correspondingly, $q$ is almost constant close to the point $[\mathrm{C}]$, and then starts to change. The similar features are also seen in a process from point $[\mathrm{A}]$ to point $[\mathrm{B}]$. Therefore, we have concluded that the variation of $q(\Delta q)$ in $H$-sweeping regardless of increasing or decreasing $H$ is essential for causing the change in the helicity domains volume fractions by driving the helicity DWs, which produce the increment of $P(\Delta P)$ from the linear $H$ variation of $P_{\text {local }}$. Furthermore, as shown in Fig. 4(d) and Fig. 4(e), the one-to-one correspondence between $\Delta P$ and $\Delta q$ can be seen also at $7 \mathrm{~K}$, although it is relatively incomprehensible compared with the results at $3 \mathrm{~K}$.

Because $P_{[110]}$ shows remarkable $T$ dependence at its emergence stage from initially un-polarized state at $10 \mathrm{~T}$ as shown in Fig. 5(a), we can check the one-to-one correspondence between $\Delta P_{[110]}$ and $\Delta q$ in the emergence stage of $P_{[110]}$. We refer to the stage in $H$-sweeping from $10 \mathrm{~T}$ (initially un-polarized state after cooling without applied $E_{p}$ ) to $9 \mathrm{~T}$ as the initial stage. In the initial stage, the change in $q$ is expected to be small. Therefore, we used the $(2 q, 2 q, 0)$-second-harmonic reflections without $E$ to accurately determine the $H$ variation of $q$, in addition to the original purpose of obtaining the absolute value of $q$. Note that the $H$ dependence of $q$ is independent of $E$ as described above. Figure 5(c) shows the typical diffraction profiles at $10 \mathrm{~T}$ and $9 \mathrm{~T}$ at $7 \mathrm{~K}$,
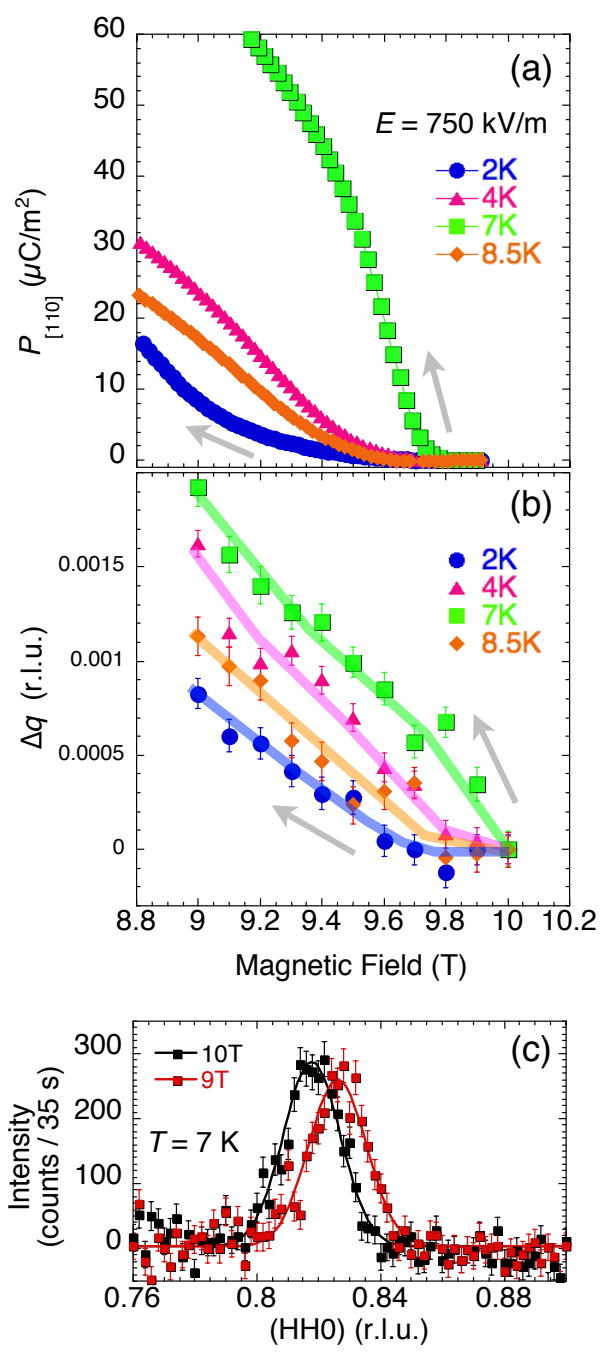

FIG. 5. (Color Online) $H$ evolution of (a) $P_{[110]}$ and (b) difference in $q(H)$ from $q(10 \mathrm{~T})(\Delta q)$, at several temperatures below $T_{\mathrm{N} 2} \sim 9 \mathrm{~K}$ after cooling at $10 \mathrm{~T}$. The solid lines are guides for the eyes. (c) Typical diffraction profiles at $10 \mathrm{~T}$ and $9 \mathrm{~T}$ at $7 \mathrm{~K}$.

and demonstrates that the $H$ variation of $2 q$ is visible despite the small intensity. Incidentally, from this experiment, we have also confirmed that the $q$ domains volume fractions are constant in $H$-sweeping by measuring the intensities of $(2 q, 2 q, 0),(-4 q, 2 q, 0)$ or $(2 q,-4 q, 0)$ reflections. They are unchanged over several cycles of $H$-sweeping (Not shown). Figure 5(b) shows the $H$ dependence of the $\Delta q$ at several $T$, which is defined here as a difference in $q(H)$ from $q(10 \mathrm{~T})$. As is apparent from Fig. 5(a) and Fig. 5(b), both the $H$ evolution of $P_{[110]}$ and the $H$ dependence of $\Delta q$ shows its maximum at around $7 \mathrm{~K}$. Moreover, both of them at $8.5 \mathrm{~K}$ are smaller than at $4 \mathrm{~K}$. These results clearly demonstrate the qualitative one-toone correspondence between the $\Delta P_{[110]}$ and $\Delta q$ of this phenomenon. 
(a)
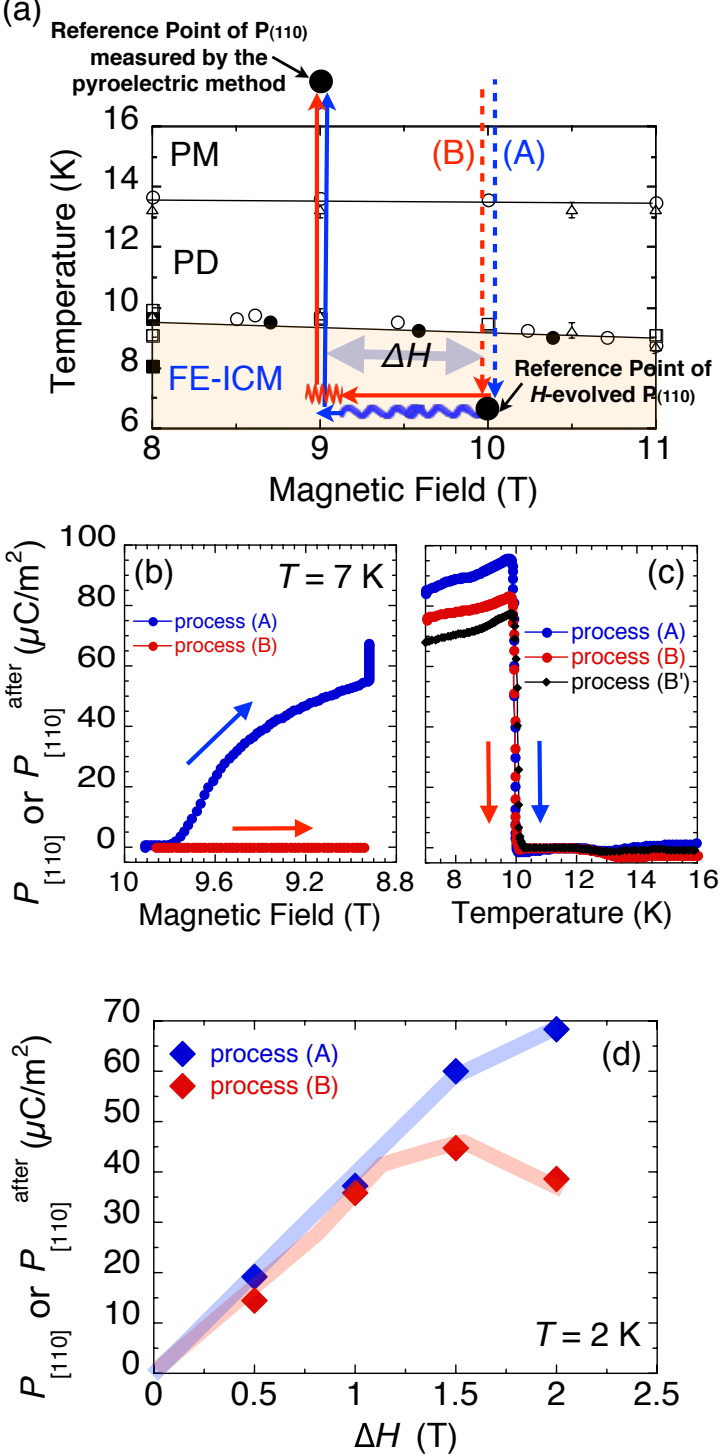

FIG. 6. (Color Online) (a) Measurement paths on the $H-T$ phase diagram for CFO. Wavy lines denote that $E$ is applied. (b) $H$ evolutions of $P_{[110]}$. (c) $T$ dependence of $P_{[110]}$ measured by the pyroelectric method in a heating run without applied $E$. In process (B') indicated by black diamond symbol in Fig. 6(c), we waited for 30 minutes after $H$-sweeping from 10 to 9 T. Then, $E$ was applied, and subsequently, $P_{[110]}^{\text {after }}$ was measured by the pyroelectric method in the heating run without applied $E$. (d) $\Delta H$ dependence of $P_{[110]}$ measured by the pyroelectric method on both paths at $2 \mathrm{~K}$. The solid lines are guide for the eyes.

\section{B. Role of $H$-sweeping and applied $E$ for the $H$ evolution of $P$}

From neutron diffraction experiments with in-situ $P_{[110]}$ measurements, we have concluded that the $H$ evolution of $P_{[110]}$ is caused by the change in the helicity domains volume fraction by driving the helicity DWs.
Although we have up to now discussed the $H$ evolution of $P_{[110]}$ for the case when we perform the $H$-sweeping under simultaneously applied $E$, the role of $E$ may be driving the helicity DWs in general, because they are ferroelectric. Then, taking into account of the one-to-one correspondence between $\Delta P_{[110]}$ and $\Delta q$, we may expect that $\Delta q$ through $H$-sweeping yields a movable state of the helicity DWs. Thus, we have investigated also the role of $H$-sweeping for the $H$ evolution of $P_{[110]}$ by the following procedure. As illustrated in Fig. 6(a), in process (A), $E$ was applied during $H$-sweeping in the same way as before, after preparation of initial state by cooling down to $7 \mathrm{~K}$ at $10 \mathrm{~T}$ without $E_{p}$. In process (B), on the other hand, $E$ was not applied during $H$-sweeping. Subsequently after $H$-sweeping from 10 to $9 \mathrm{~T}(\Delta H=1 \mathrm{~T})$, $E$ was applied for 5 minutes at $9 \mathrm{~T}$. Hereafter, we refer to the $P_{[110]}$ induced by applying $E$ "after" $H$-sweeping in process (B) as " $P_{[110]}^{\text {after" }}$. It should be noted that $P_{[110]}^{\text {after }}$ can not be estimated by time integration of polarization current induced by applying $E$ after $H$-sweeping, because huge currents (higher by two orders than signal polarization currents) due to the sample behaving like a capacitor have flown at application or removal of $E$ at $9 \mathrm{~T}$. Therefore, we have estimated $P_{[110]}^{\text {after }}$ by the pyroelectric method in a heating run without applied $E$. Figure 6(b) shows the $H$ evolution of $P_{[110]}$ in both processes. No emergence of $P_{[110]}$ has been observed in process $(\mathrm{B})$, indicating that " $E$ drives the DWs" as expected. Figure 6(c) shows the $T$ dependence of $P_{[110]}$ in process $(\mathrm{A})$ and $P_{[110]}^{\text {after }}$ in process (B) measured by the pyroelectric method in the heating run without applied $E$. Note that as shown in Fig. 6(a), a reference point of the $H$-evolved $P_{[110]}$ in the time integration process is at $10 \mathrm{~T}$ and $7 \mathrm{~K}$ in the FE-ICM phase immediately after cooling, whereas, that of $P_{[110]}$ and $P_{[110]}^{\text {after }}$ measured with the pyroelectric method in the heating run is at $9 \mathrm{~T}$ and $18 \mathrm{~K}$ in $\mathrm{PM}(\mathrm{PE})$ phase. As shown in Fig. 6(b) and Fig. 6(c), the value of $P_{[110]}$ measured by the pyroelectric method in process $(\mathrm{A})$ is almost equivalent to that of $H$-evolved $P_{[110]}$ in process (A), despite different reference points. This result validates that we can compare the value of $P_{[110]}$ or $P_{[110]}^{\text {after having differ- }}$ ent reference point, and that application or removal of $E$ does not affect the value of $P_{[110]}$ or $P_{[110]}^{\text {after }}$. As shown in Fig. $6(\mathrm{c}), P_{[110]}^{\text {after }}$ at $7 \mathrm{~K}$ is comparable with $P_{[110]}$ at $7 \mathrm{~K}$ in process $(\AA)$. This result indicates that the $H$ variation of $q$ yields a movable state of the helicity DWs and $P_{[110]}^{\text {after }}$ is induced by applying $E$ after $H$-sweeping via driving the helicity DWs. Therefore, simultaneous application of $E$ and $H$-sweeping is not necessary for this phenomenon ; $\Delta q$ through $H$-sweeping cause the helicity DWs to be the movable state, and then $E$ drives them.

Furthermore, we have obtained distinctive results, which suggest that the ferroelectric helicity DWs are not simply dragged by the applied electric field. In process (B'), after preparation of initial state by cooling down to $7 \mathrm{~K}$ at $10 \mathrm{~T}$ without $E_{p}$, we have performed $H$-sweeping 
from 10 to $9 \mathrm{~T}$ without applied $E$ at $7 \mathrm{~K}$, which is the same as process (B) so far. Then, we have waited for 30 minutes before $E$ is applied for 5 minutes at 9 T. Subsequently, we have measured the pyroelectric current in the heating run without applied $E$ as the same as process (B). As a result, we find a finite $P_{[110]}^{\text {after }}$, as shown in Fig. $6(\mathrm{c})$ (process $\left(\mathrm{B}^{\prime}\right)$ ). This result suggests that the movable state of the helicity DWs is conserved for a while after $H$-sweeping. Moreover, we find a novel $\Delta H$ dependence of $P_{[110]}$ or $P_{[110]}^{\text {after }}$ at $2 \mathrm{~K}$, as measured by the pyroelectric method. As shown in Fig. 6(d), $P_{[110]}^{\text {after }}$ is comparable with $P_{[110]}$ in process (A) up to $\Delta H=1 \mathrm{~T}$, but above $1 \mathrm{~T}, P_{[110]}^{\text {after }}$ starts to deviate from the $\Delta H$ dependence of $P_{[110]}$ in process $(\mathrm{A})$. Furthermore, when $\Delta H$ exceeds 1.5 $\mathrm{T}, P_{[110]}^{\text {after }}$ decreases with increase $\Delta H$. Such $\Delta H$ dependence of $P_{[110]}$ or $P_{[110]}^{\text {after }}$ have also been confirmed at $7 \mathrm{~K}$ (Not shown). In the following section, we will propose a model of this phenomenon, which are taking into account these distinctive results.

\section{Relaxation process in the $H$ evolution of $P$}

Figure 6(b) also suggests the existence of a relaxation process in this phenomenon, because $P_{[110]}$ measured in process $(\mathrm{A})$ increases $\sim 15 \mu \mathrm{C} / \mathrm{m}^{2}$ at $9 \mathrm{~T}$ even after stoping $H$-sweeping. To elucidate more precisely an elementary process, we have performed measurements of $P_{[110]}$ by step-by-step $H$-sweeping with $H$ steps of 0.5 $\mathrm{T}(\delta H=0.5 \mathrm{~T})$ under applied $E$ instead of continuous $H$-sweeping. As shown in Fig. 7(a), the overall $H$ evolution of $P_{[110]}$ measured with both procedures are almost similar, as also described in previous section, except for the increment of $P_{[110]}$ by the amount of relaxation process after stoping $H$-sweeping in the step-by-step measurement. Figure 7(b) shows a part of this process as a function of time. As is clearly seen in Fig. $7(\mathrm{~b}), P_{[110]}$ gradually increases even after stoping $H$-sweeping, and is relaxed over $1 \sim 2$ minutes. This relatively long relaxation time corroborates our conclusion that this phenomenon is caused by the change not in the magnetic structure but in the domain structure. Note that this relaxation time is almost independent of $T$ and $H$-sweeping rate in a range of $3.07 \sim 11.4 \mathrm{mT} / \mathrm{s}$. In addition, the $H$ evolution of $P_{[110]}$ does not occur until $\delta H$ exceeds $\sim 0.2$ $\mathrm{T}$ after starting $H$-sweeping (see Fig. $7(\mathrm{~b})$ ). This result suggests that the $H$ evolution of $P_{[110]}$ has a threshold $\delta H$ on emergence. However, we do not ascertain this feature to be a part of helicity DW dynamics, because both fields were applied simultaneously and hence both fields intricately affected the $H$ evolution of $P_{[110]}$. Further study on helicity DW dynamics is necessary to clarify this issue.
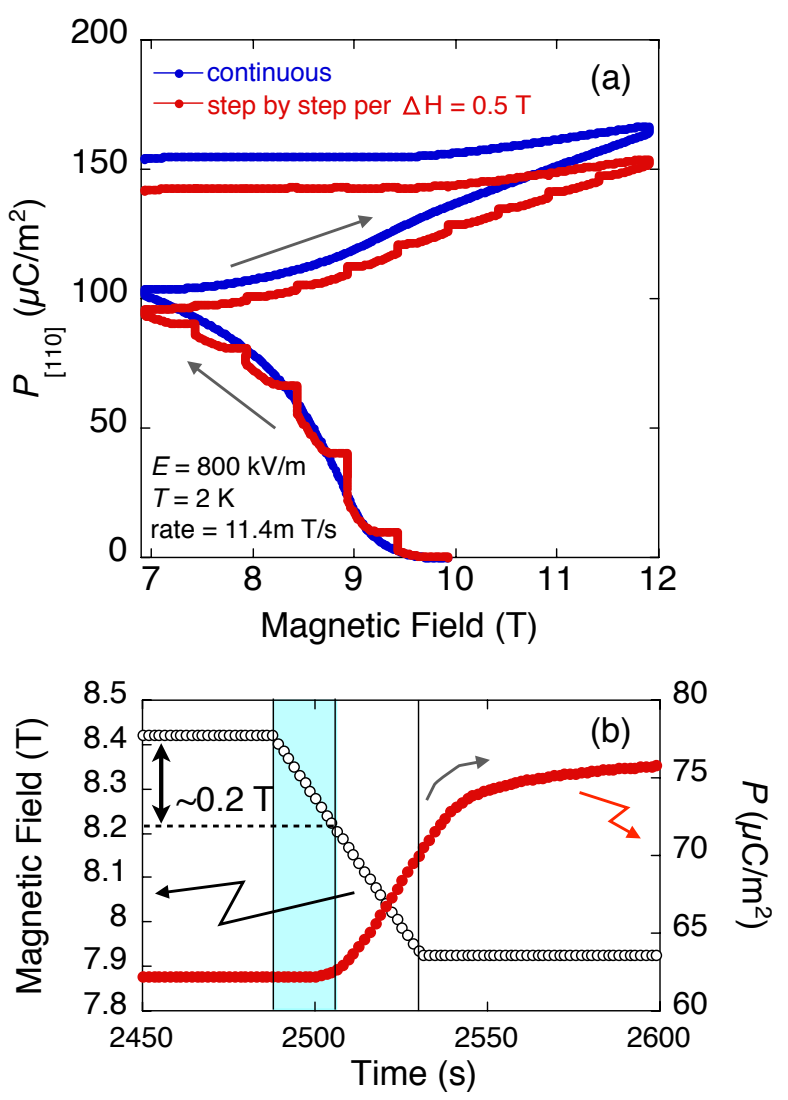

FIG. 7. (Color Online) (a) $H$ dependence of $P_{[110]}$ for two kinds of $H$-sweeping procedures under applied $E$. (b) Typical $H$ evolution of $P_{[110]}$ by step-by-step $H$-sweeping with $H$ steps of $0.5 \mathrm{~T}$ as a function of time.

D. Temperature dependence of the $H$ evolution of $P$ in a late stage over several cycles of $H$-sweeping

Figures $8(\mathrm{a})-8(\mathrm{c})$ show the $H$ evolution of $P_{[110]}$ when we have performed $H$-sweeping over several cycles under applied $E$ of $600 \mathrm{kV} / \mathrm{m}$ at $2 \mathrm{~K}, 7 \mathrm{~K}$ and $8.5 \mathrm{~K}$, respectively. ${ }^{27}$ In an early stage of up to a quarter cycle of $H$-sweeping beyond the initial stage, we have found that overall $H$ evolution of $P_{[110]}$ is almost the same, although we can see the remarkable $T$ dependence of $P_{[110]}$ corresponding to the $H$ variation of $q$ in the initial stage as shown in Fig. 5(b). In a late stage over several cycles of $H$-sweeping, in contrast, the induced value of $P_{[110]}$ clearly depends on $T: P_{[110]}$ in the late stage at $2 \mathrm{~K}$ is almost saturated at less than a half value obtained by proper poling. With increasing $T, P_{[110]}$ in the late stage increases toward the value obtained by proper poling. Especially at $8.5 \mathrm{~K}$, it is the comparable to the value obtained by proper poling.

As shown in Figs. 4(b) and 4(e), overall trajectory of $q(H)$ at $2 \mathrm{~K}$ and $7 \mathrm{~K}$ are the almost same except for the initial stage. Moreover, the trajectory of $q(H)$ over several cycles of $H$-sweeping is unchanged. Therefore, recalling that the $H$ variation of $\Delta q$ leads to the increment 


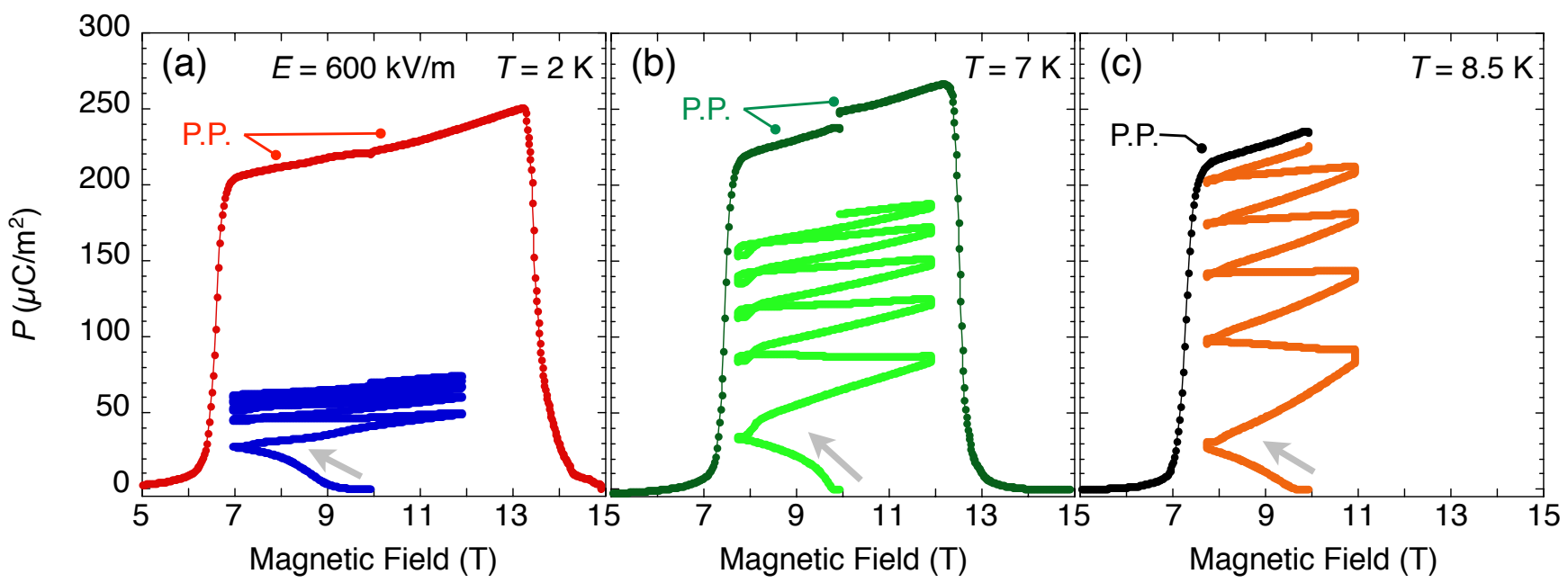

FIG. 8. (Color Online) [(a)-(c)] $H$ evolution of $P_{[110]}$ over several cycles of $H$-sweeping under applied $E$ at (a) 2 K, (b) $7 \mathrm{~K}$ and (c) $8.5 \mathrm{~K}$. For comparison, the $H$ dependence of $P_{[110]}$ measured with proper poling (P.P.) procedure is also plotted.

of $P_{[110]}$ by the change in the helicity domains volume fractions as mentioned in Sec. III A, we can expect that $P_{[110]}$ should keep on increasing in the late stage over several cycles of $H$-sweeping under applied $E$ in any $T$ so as to realize the single helicity domain state, which can be obtained by proper poling. In spite of such expectation, the result shown in Fig. 8(a) indicates that some suppression factor exists, which prevents the helicity DWs from further moving. In the following section, this anomalous $T$ dependence of $P_{[110]}$ in the late stage will be discussed.

\section{E. Model Proposal}

In Fig. 9(a), we show a schematic illustration of the $q$ domain and the helicity domain structures in the FEICM phase. As mentioned in Sec. III A, the $H$ evolution of $P_{[110]}$ is caused by the change in the helicity domains volume fraction by driving the helicity DWs. When the helicity DWs are driven by $H$-sweeping under applied $E$, a ratio of a sign of the electric charges on the electrode surfaces is modified as shown in Fig. 9(b). This would be the macroscopic picture on the $H$ evolution of $P_{[110]}$.

Here, we move on to a microscopic picture of helicity DWs motion. Among our experimental results, especially the following two results suggest that microscopic helicity DWs motion is not simple, compared with the usual ferroelectric motion, in which they are simply dragged by the applied electric field conjugating to the ferroelectric polarization.

1. The value of evolved $P_{[110]}$ is determined by the variation of $q, \Delta q$. (Sec. III A)

2. After $H$-sweeping without applied $E$, the movable state of the helicity DWs is conserved over several tens of minutes until $E$ is applied. (Sec. III B)
In the following, we consider the microscopic picture of the helicity DWs movement in accordance with these distinctive experimental results.

Judging from the result 2, we can expect that the variation of $q, \Delta q$, in $H$-sweeping changes a state of the helicity domains and the helicity DWs separating the righthanded and the left-handed helical domains. Now, we consider how $\Delta q$ affects the helicity domains and the helicity DWs. In the conventional DWs in Heisenberg ferromagnets, magnetic moments gradually rotate like Bloch wall to locally minimize the energy loss of ferromagnetic exchange interaction between neighbor spins. In the case of the helicity DWs in the FE-ICM phase, in contrast, it may be relatively difficult to achieve the local energy minimization between neighbor spins around the helicity DW, because the changeover from one helicity domain to the other helicity one by gradual rotation is impossible (Fig. 10(a)(I)). In $H$-sweeping, $\Delta q$ would cause the magnetic moments a global rotation so as to uniformly change a relative angle by amount of $2 \pi \Delta q$ between neighbor spins. If boundaries of the helical arrangement is open, the relative angle change would be easily achieved. In the helical spin arrangement in the FE-ICM phase, however, the helicity DWs, namely boundaries of the helicity domain, would work as "pinning center" for the global rotations due to the impossible changeover in the helicity DWs, which would prevents a neighborhood of the helicity DWs from achieving the relative angle change. As a result, the global rotations should mainly affect the neighborhood of the helicity DWs. Taking into consideration the difficulty of the local energy minimization around the helicity DW, these region around the helicity DWs would store an extra exchange energy as "twist" due to the global rotations. We refer to such extra exchange energy stored region as an affected region. From the result 1 , we expect that an affected region volume is proportional to $\Delta q$ to a certain extent. 


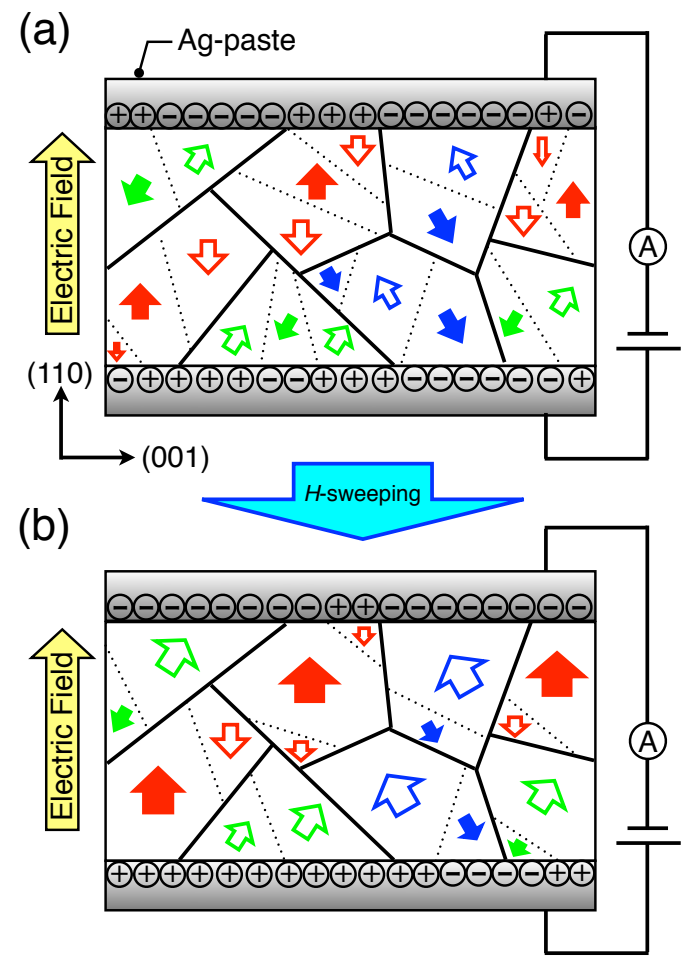

FIG. 9. (Color Online) Schematic illustration of (a) the macroscopic $q$ domain and the helicity domain structures with $P$-induced charge on the electrode surfaces in the FE-ICM phase and (b) the $q$ domain and the helicity domain structures after driving the helicity DWs by $H$-sweeping and applied $E$. Opaque (transparent) red, green, and blue arrows represent the $P$ induced by the left-handed (right-handed) helical magnetic order in (110), $(\overline{2} 10)$ and $(1 \overline{2} 0)$ domain, respectively. Solid lines and dashed lines denote the $q$ domain walls and the helicity domain walls, respectively. Note that the size of arrows denoting $P$ has no deeper meaning.

Here, we consider how much the global rotation affects the neighborhood of the helicity DWs, using neutron diffraction profiles. There would be an extreme case that the affected regions are perfectly disordered by the global rotation. In this case, reduction of the fundamental magnetic Bragg reflection should be detected, because the helicity domain volume is reduced due to the (disordered) affected region. To quantitatively evaluate the reduction of the intensity, we roughly estimated the volume fraction of the affected region for the case of $\Delta H=2.5$ $\mathrm{T}$ from initial state (from $10 \mathrm{~T}$ to $7.5 \mathrm{~T}$ ) at $2 \mathrm{~K}$ through $H$-sweeping without applied $E$, on the assumption that $P_{[110]}^{\text {after }} / P_{[110]}^{P . P_{i}}$ is proportional to the volume fraction of the affected region. For the estimation, we have used the result shown in Fig. $6(\mathrm{~d})\left(P_{[110]}^{\text {after }}(\Delta H=2.5 \mathrm{~T}) \sim 25 \mu \mathrm{C} / \mathrm{m}^{2}\right)$ and the value of $P_{[110]}^{P . P} \sim 220 \mu \mathrm{C} / \mathrm{m}^{2}$ in the single helicity domain state obtained by proper poling. According to the rough estimation, the disordered affected regions account for $\sim 10 \%$ of the sample for that case. As shown (a)

(I)

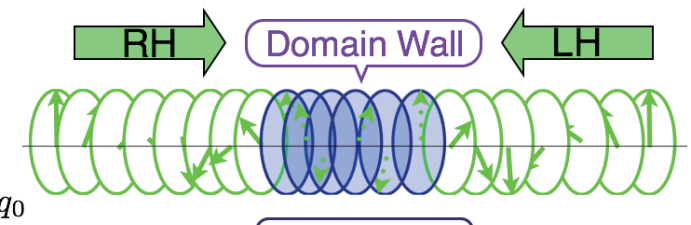

(II)

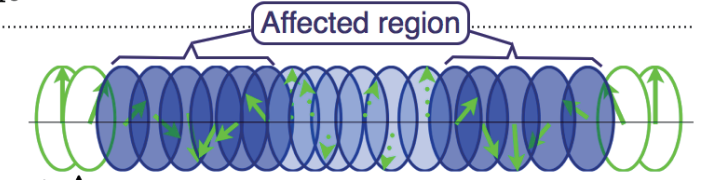

$q_{0}+\Delta q$

(III)

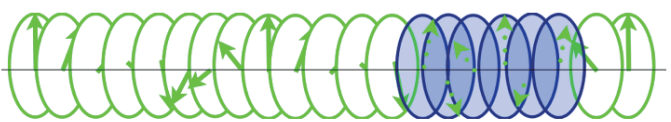

$\stackrel{\text { (oo1) }}{\longrightarrow} q_{0}+\Delta q \quad$ Electric Field

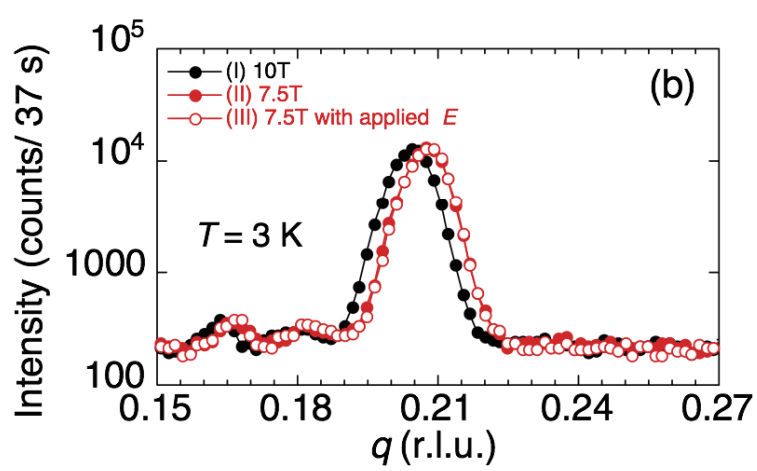

FIG. 10. (Color Online) (a) Schematic illustration of the helicity DW separating RH- and LH- helicity domains. (I) Unpolarized state immediately after cooling without $E_{p}$. A right blue shaded area demonstrates the helicity DW. (II) By the global rotations, the extra exchange energy would be stored in the affected region. A dark shaded areas demonstrate the affected regions. (III) Then, the affected regions and the helicity DWs would be reconstructed by applied $E$ so as to enlarge the helicity domain favored by the direction of $E$. (b) Typical diffraction profiles on a logarithmic scale at $10 \mathrm{~T}$ or $7.5 \mathrm{~T}$ with or without applied $E$. Indices of $(\mathrm{I}) \sim(\mathrm{III})$ correspond to the domain states illustrated in FIG. 10(a).

in Fig. 3(a) (the diffraction profiles for existence/absence of the affected region should correspond to the profile at $7.5 \mathrm{~T}$ without/with $E$, respectively), however, the reduction of the intensity by $\sim 10 \%$ is not observed within poor resolution of this experiment. This result indicates that the affected regions are not perfectly disordered.

Similarly, it is possible that, although the neighborhood of the helicity DWs are disturbed by the global rotation, the affected regions have some short range order, which should give rise to diffusive scattering superposed on the fundamental magnetic Bragg reflection with $q$. Figure 10(b) shows typical diffraction profiles on a logarithmic scale at $10 \mathrm{~T}$ or $7.5 \mathrm{~T}$ with or without applied 
$E$. The diffusive scattering is not observed as well within this experimental resolution. Thus, neutron diffraction profiles shown in Fig. 3(a) and Fig. 10(b) suggest that the affected regions keep the original helical correlation. In considering the model of the microscopic helicity DWs movement, we should also take this result into account;

3. The affected regions keep the original helical correlation.

Taking into account the distinctive results $1 \sim 3$, we have depicted the helicity DWs movement as follows: the global rotations generate the affected regions, whose volume should be proportional to $\Delta q$ to a certain extent, while the affected regions keep the original helical correlation (Fig. 10(a)(II)). Because the affected regions would store the extra exchange energy as "twist", they should be sensible for $E$. Then, the affected regions and the helicity DWs would be reconstructed by applied $E$ so as to enlarge the helicity domain favored by the direction of $E$ (Fig. 10(a)(III)). As a result, the helicity DWs movement is achieved.

Based on this naive model, we discuss the other results. (1) The anomalous $T$ dependence of $P_{[110]}$ (Sec. III D): in the process of the generation of the affected region and their reconstruction by several cycles of $H$-sweeping under applied $E$, we can assume that the affected regions partially remain without being reconstructed and behave as "defect" preventing the helicity DWs from further moving. Actually, $P_{[110]}$ in the late stage at $2 \mathrm{~K}$ is almost saturated at less than a half value obtained by proper poling, as described in Sec. III D. However, thermal fluctuation may assist the reconstruction of the helicity domains by driving the helicity DWs, and thus, especially at $8.5 \mathrm{~K}$, the almost single helicity domain state is realized. (2) Following our proposed model, we can expect that the $H$ evolution of $P_{[110]}$ is independent of $H$-sweep rate, because crucial factor for $H$ evolution of $P_{[110]}$ is $\Delta q$. Actually, as shown in Fig. 2(c), we have confirmed that the $H$ evolution of $P_{[110]}$ is not sensitive to $H$-sweep rate in a range of $3.07 \sim 11.4 \mathrm{mT} / \mathrm{s}$. (3) Our proposed model does not quantitatively clarify the relaxation process or the threshold $\delta H$ on emergence of $P$ (Sec. III C). Moreover, the model does not include why the helicity DWs become sensible for $E$ through the stored extra exchange energy as "twist" and how the helicity DWs move. This point would be concerned with

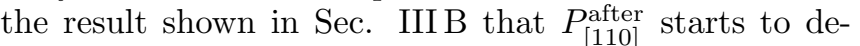
viate from the $\Delta H$ dependence of $P_{[110]}$ in process (A) above $1 \mathrm{~T}$; why $E$ does not thoroughly reconstruct the affected region produced by $\Delta H$ above $1 \mathrm{~T}$ without applied $E$. To establish this model, direct observation of the helicity DWs motion process, and theoretical calculation of the helicity DWs motion based on the microscopic spin dynamics in the affected region are highly desirable.

Finally, we emphasize the following two points. First, what we have controlled is not "the nuclei growth" of one of the helicity domain by applied $E$ on the ferroelectric transition ${ }^{22}$ but "the reconstruction" of the helicity domain by driving the helicity DWs, which are not originally driven by only applied $E$ due to the large coercive electric field. Besides providing a new technique of the helicity DW control in CFO systems, the control of the helicity domain in the present study is expected to be realized in other spin-driven ferroelectric materials having a large coercive electric field in a multiferroic phase. Second, the present study is nothing less than achievement of driving antiferromagnetic DW as well as that of driving the frozen ferroelectric DW even at $2 \mathrm{~K}$. As F. Kagawa et al. have pointed out, ${ }^{9}$ in conventional antiferromagnets with a magnetic order of magnetic modulation wave vector $\boldsymbol{Q}$, it is difficult to experimentally drive antiferromagnetic DWs, because it requires spatially modulated magnetic field matching $\boldsymbol{Q}$. In contrast, we can easily drive antiferromagnetic DWs in multiferroics by applying an uniform electric filed through the cross-correlation between antiferromagnetism and ferroelectricity. Therefore, multiferroics also provide a good opportunity to investigate antiferromagnetic DWs and their dynamics.

\section{SUMMARY}

We have found a peculiar irreversible evolution of $P_{[110]}$ by $H$-sweeping under applied $E$ in the FE-ICM phase of a spin-driven ferroelectric $\mathrm{CFO}$, despite the large coercive electric field above $1 \mathrm{MV} / \mathrm{m}$ in this phase. Using neutron diffraction experiments with in-situ $P_{[110]}$ measurements and the off-bench $P$ measurements, we have revealed that the variation of $q, \Delta q$, in $H$-sweeping regardless of increasing or decreasing $H$ leads to the increment of $P_{[110]}$, which is achieved by the change in the helicity domains volume fractions by driving the helicity DW. From the further off-bench $P_{[110]}$ measurements, we have specified the role of $H$-sweeping and applied $E$ : $\Delta q$ by $H$-sweeping yields a movable state of the helicity DWs, and applied $E$ drives them.

Based on these results, we propose a simple model for this phenomenon, which is expected to be realized in other spin-driven ferroelectric materials as well. The variation of $q$ would generate the affected regions, which may store the extra exchange energy as "twist" and should be sensible for $E$, while the affected regions keep the original helical correlation. Then, the affected regions and the helicity DWs would be reconstructed by applied $E$ so as to enlarge the helicity domain favored by the direction of $E$. Although this naive model does not clarify the some results such as the helicity DWs dynamics, it can account for the some other distinctive behavior of $P_{[110]}$. To establish our proposal model, further study on helicity DW dynamics, direct observation of the helicity DWs motion process, and theoretical calculation of the helicity DWs motion based on the microscopic spin dynamics in the affected region are highly desirable.

The present study demonstrates that the magnetoelectric cross correlation in driving multiferroic DW: we could activate the frozen ferroelectric DW by assistance 
of $H$-sweeping. This is also an achievement of driving antiferromagnetic DW by applied uniform $E$, which is difficult in conventional antiferromagnets in principle.

\section{ACKNOWLEDGMENTS}

We thank Late Group Leader of Nano Physics Group (NIMS), Tadashi Takamasu, for pyroelectric measure- ments using the $15 \mathrm{~T}$ magnet at NIMS in the early stage of our study. We also thank HZB for the allocation of neutron beamtime. The neutron diffraction experiments at HZB were carried out according to Proposal Nos. PHY01-2963 and PHY-01-3270. This work was supported by a Grant-in-Aid for Scientific Research (C) (Grant Nos. 23540424 and 26400369) from Japan Society for the Promotion of Science.
* E-mail address: tamatsukuri@nsmsmac4.ph.kagu.tus.ac.jp

1 T. Kimura, T. Goto, H. Shintani, K. Ishizaka, T. Arima, and Y. Tokura, Nature (London) 426, 55 (2003).

${ }^{2}$ W. Eerenstein, N. D. Mathur, and J. F. Scott, Nature (London) 442, 759 (2006).

3 T. Kimura and Y. Tokura, J. Phys.: Condens. Matter 20, 434204 (2008).

${ }^{4}$ Y. Tokura and S. Seki, Adv. Mater. 22, 1554 (2010).

${ }^{5}$ H. Katsura, N. Nagaosa, and A. V. Balatsky, Phys. Rev. Lett. 95, 057205 (2005).

${ }^{6}$ C. Jia, S. Onoda, N. Nagaosa, and J. H. Han, Phys. Rev. B 76, 144424 (2007).

7 T. Arima, J. Phys. Soc. Jpn. 76, 073702 (2007).

${ }^{8}$ F. Kagawa, M. Mochizuki, Y. Onose, H. Murakawa, Y. Kaneko, N. Furukawa, and Y. Tokura, Phys. Rev. Lett. 102, 057604 (2009).

${ }^{9}$ F. Kagawa, Y. Onose, Y. Kaneko, and Y. Tokura, Phys. Rev. B 83, 054413 (2011).

10 M. Gajek, M. Bibes, S. Fusil, K. Bouzehouane, J. Fontcuberta, A. Barthelemy, and A. Fert, Nature Materials 6, 296 (2007).

11 S. Mitsuda, N. Kasahara, T. Uno, and M. Mase, J. Phys. Soc. Jpn. 67, 4026 (1998).

12 S. Mitsuda, M. Mase, K. Prokes, H. Kitazawa, and H. A. Katori, J. Phys. Soc. Jpn. 69, 3513 (2000).

13 T. Nakajima, S. Mitsuda, S. Kanetsuki, K. Prokes, A. Podlesnyak, H. Kimura, and Y. Noda, J. Phys. Soc. Jpn. 76, 043709 (2007).

14 S. Seki, Y. Yamasaki, Y. Shiomi, S. Iguchi, Y. Onose, and Y. Tokura, Phys. Rev. B 75, 100403(R) (2007).

15 S. Kanetsuki, S. Mitsuda, T. Nakajima, D. Anazawa, H. A. Katori, and K. Prokes, J. Phys.: Condens. Matter 19, 145244 (2007)

16 N. Terada, T. Nakajima, S. Mitsuda, H. Kitazawa, K. Kaneko, and N. Metoki, Phys. Rev. B 78, 014101 (2008).

17 E. Pachoud, C. Martina, B. Kundys, C. Simona, and A. Maignana, J. Solid State Chem. 183, 344 (2010).

18 T. Kimura, J. C. Lashley, and A. P. Ramirez, Phys. Rev. B 73, 220401(R) (2006)

19 T. Nakajima, S. Mitsuda, S. Kanetsuki, K. Tanaka, K. Fu- jii, N. Terada, M. Soda, M. Matsuura, and K. Hirota, Phys. Rev. B 77, 052401 (2008).

20 T. Nakajima, S. Mitsuda, T. Nakamura, H. Ishii, T. Haku, Y. Honma, M. Kosaka, N. Aso, and Y. Uwatoko, Phys. Rev. B 83, 220101 (2011).

${ }^{21}$ S. Seki, H. Murakawa, Y. Onose, and Y. Tokura, Phys. Rev. Lett. 103, 237601 (2009).

22 T. Nakajima, S. Mitsuda, K. Takahashi, H. Yamazaki, K. Yoshitomi, M. Soda, M. Matsuura, and K. Hirota, Phys. Rev. B 82, 064418 (2010).

${ }^{23}$ T. R. Zhao, M. Hasegawa, and H. Takei, J. Cryst. Growth 166, 408 (1996).

24 N. Terada, S. Mitsuda, K. Prokeš, O. Suzuki, H. Kitazawa, and H. A. Katori, Phys. Rev. B 70, 174412 (2004).

25 For clarity, we present the data obtained by $2 q$ measurements in this work to Fig. 2(b), because the data of $H$ dependence of $q$ reported by S. Mitsuda et al. in Ref. 12 was measured at $7 \mathrm{~K}$ and across the phase boundary between the FE-ICM phase and the 5SL phase.

26 T. Nakajima, S. Mitsuda, K. Takahashi, M. Yamano, K. Masuda, H. Yamazaki, K. Prokes, K. Kiefer, S. Gerischer, N. Terada, H. Kitazawa, M. Matsuda, K. Kakurai, H. Kimura, Y. Noda, M. Soda, M. Matsuura, and K. Hirota, Phys. Rev. B 79, 214423 (2009).

27 As was reported by T. Nakajima et al. ${ }^{20}$ and S. Mitsuda et $a l .,{ }^{28}$ the $q$ domains volume fraction is easily changed by applying only $5 \sim 10 \mathrm{MPa}$ of uniaxial pressure at room temperature. Therefore, the reason why the value of $P_{[110]}$ at $2 \mathrm{~K}$ in Fig. $8(\mathrm{a})$ is different from that of $P_{[110]}$ at 2 $\mathrm{K}$ in Fig. 2(c) is attributed to the difference of not the magnitude of applied $E$ but the $q$ domains volume fraction owing to slight difference of sample mounting with varnish. Within the same experiment period, however, the value of $P_{[110]}$ is reproducible and thus it is considered that the $q$ domains volume fraction is unchanged.

28 S. Mitsuda, K. Yoshitomi, T. Nakajima, C. Kaneko, H. Yamazaki, M. Kosaka, N. Aso, Y. Uwatoko, Y. Noda, M. Matsuura, N. Terada, S. Wakimoto, M. Takeda, and K. Kakurai, J. Phys.: Conf. Ser. 340, 012062 (2012). 